\title{
ANTIPHOSPHOLIPID ANTIBODIES AND MULTIPLE ORGAN FAILURE IN CRITICALLY ILL CANCER PATIENTS
}

\author{
Jorge I. F. Salluh, ${ }^{\mathrm{I}, \mathrm{II}}$ Márcio Soares, ${ }^{\mathrm{I}}$ Ernesto De Meis, ${ }^{\mathrm{III}, \text { IV }}$
}

doi: $10.1590 / \mathrm{S} 1807-59322009000200003$

Salluh JIF, Soares M, De Meis E. Antiphospholipid antibodies and multiple organ failure in critically ill cancer patients. Clinics. 2009;64:79-82.

OBJECTIVES: To describe the clinical outcomes and thrombotic events in a series of critically ill cancer patients positive for antiphospholipid (aPL) antibodies.

DESIGN: Retrospective case series study.

SETTING: Medical-surgical oncologic intensive care unit (ICU).

Patients and Participants: Eighteen patients with SIRS/sepsis and multiple organ failure (MOF) and positive for aPL antibodies, included over a 10-month period.

INTERVENTIONS: None

MEASUREMENTS AND RESULTS: aPL antibodies and coagulation parameters were measured up to 48 hours after the occurrence of acrocyanosis or arterial/venous thrombotic events. When current criteria for the diagnosis of aPL syndrome were applied, 16 patients met the criteria for "probable" and two patients had a definite diagnosis of APL syndrome in its catastrophic form (CAPS). Acrocyanosis, arterial events and venous thrombosis were present in eighteen, nine and five patients, respectively. Sepsis, cancer and major surgery were the main precipitating factors. All patients developed MOF during the ICU stay, with a hospital mortality rate of $72 \%$ (13/18). Five patients were discharged from the hospital. There were three survivors at 90 days of follow-up. New measurements of lupus anticoagulant (LAC) antibodies were performed in these three survivors and one patient still tested positive for these antibodies.

CONCLUSIONS: In this small series of patients, we observed a high frequency of auto-antibodies and micro- and macro-vascular thrombotic events in critically ill cancer patients. The coexistence of sepsis or SIRS and aPL antibodies was often associated with MOF and death. More studies are necessary to determine the pathophysiological significance of antiphospholipid antibodies in severely ill cancer patients.

KEYWORDS: Antiphospholipid syndrome; Multiple organ failure; Cancer; Thrombosis; Sepsis; Lupus anticoagulant.

\section{INTRODUCTION}

Antiphospholipid (aPL) syndrome is a disorder characterized by recurrent venous or arterial thrombosis

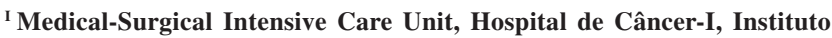
Nacional de Câncer, Rio de Janeiro, Brazil.

II Immunopharmacology laboratory, IOC, Fundação Oswaldo Cruz, Rio de Janeiro, Brazil

III Hematology Service, Instituto Nacional de Câncer, Rio de Janeiro, Brazil.

Iv Clinical Pathology Service, Instituto Nacional de Câncer, Rio de Janeiro, Brazil.

Email: jorgesalluh@yahoo.com.br, jsalluh@inca.gov.br

Tel.: 5521 2506-6120 Fax: 5521 2294-8620

Received for publication on September 08, 2008

Accepted for publication on October 08, 2008
}

associated with distinctive laboratory abnormalities that include elevated levels of antibodies directed against membrane phospholipids (i.e., anticardiolipin [aCL] antibody, antiphosphatidylserine), antibodies associated with plasma proteins (predominantly beta-2 glycoprotein I), or evidence of a circulating anticoagulant (the lupus anticoagulant). ${ }^{1}$ The presence of aPL antibodies has been described in cancer patients ${ }^{1}$ and was recently associated with an increased rate of thrombosis and a worse prognosis..$^{2,3}$ In cancer patients, aPL autoantibodies may represent a detectable link and a marker of crosstalk between the coagulation and immune systems. The catastrophic aPL syndrome (CAPS, Asherson's syndrome) 
is considered a rare and severe form of aPL syndrome, often associated with multiple organ failure (MOF) and death. ${ }^{4}$ This autoimmune response is described in many pathologic situations, including infections ${ }^{5}$ and cancer, ${ }^{3}$ and results in the emergence of autoantibodies, such as aPL, in a permanent or transient manner. ${ }^{6}$ Cancer patients are frequently exposed to severe septic shock and systemic inflammatory response syndrome (SIRS). ${ }^{7}$ In acute severe illnesses like sepsis, major surgery and SIRS, the immune response is frequently implicated in the occurrence of coagulation abnormalities. ${ }^{8}$ However, the literature to date shows very scarce data on the association of apL autoantibodies with the occurrence of life-threatening thrombotic events and multiorgan failure in cancer patients.

The aim of the present study was to describe the clinical picture and the outcomes in a series of critically ill cancer patients positive for aPL antibodies.

\section{METHODS AND PATIENTS}

This was a retrospective study performed at the medicalsurgical intensive care unit (ICU) of the Instituto Nacional de Câncer, a multidisciplinary cancer center in Brazil. Information on the organization and policies of our ICU is described elsewhere. ${ }^{7}$ Our study was approved by the Institutional Review Board.

We reviewed the charts and medical records of adult (age $\geq 18$ years) patients with a definite diagnosis of cancer and positive aPL antibodies, who had been admitted to the ICU between December 2006 and September 2007. Demographic, clinical, laboratory and outcome data were collected. Active cancer was defined as a recent diagnosis, or by the presence of clinical and radiologic signs of activity, or as any patients undergoing treatment at the time of our study (current chemotherapy or radiation therapy). The Simplified Acute Physiology Score (SAPS) II was calculated at ICU admission'. Sepsis was diagnosed according to the ACCP/ SCCM criteria. ${ }^{10}$

CAPS was diagnosed according to current definitions. ${ }^{11}$ aPL antibodies were detected by ELISA (Anticardiolipin, standardized assay, consistent with manufacturer's instructions) and clotting assay (lupus anticoagulant using the Russel's Viper venom method). D-dimer, antithrombin, protein $\mathrm{C}$, platelet count, prothrombin time, activated partial thromboplastin time and fibrinogen levels were determined using clotting assays (Dade Diagnostics, USA). aPL antibodies and coagulation parameters were measured up to 48 hours after the occurrence of acrocyanosis or arterial/ venous thrombosis in all patients. DVT was diagnosed with doppler and US, and gastric ischemia was diagnosed using endoscopy.
The decision to treat patients with corticosteroids, plasmapheresis or heparin was at the discretion of the assistant intensive care physicians and hematologists.

Standard descriptive statistics were used. Data are presented as median (interquartile range $25 \%-75 \%$ ) or median (range).

\section{RESULTS}

During the study period, a total of 540 patients were admitted to the ICU. Only 18 (male $=13$, female $=5)$ tested positive for apL antibodies during their ICU stay; these individuals were included in the present study. The cancer types represented across these 18 patients included: gastrointestinal $(n=7)$, larynx $(n=4)$, non-Hodgkin's lymphoma $(n=3)$, skin/soft tissue $(n=2)$, multiple myeloma $(\mathrm{n}=1)$ and urinary bladder $(\mathrm{n}=1)$. Most of the patients had an active malignancy (15/18). Patient group characteristics are shown in Table 1. All the patients fulfilled the SIRS criteria and had elevated plasma $\mathrm{C}$-reactive protein concentrations on the day of diagnosis of $17.3(13-25.2) \mathrm{mg} / \mathrm{dL}$ (median, $25 \%-75 \%$ interquartile range).

Table 1 - Patient characteristics

\begin{tabular}{|c|c|}
\hline Variables & All patients $(\mathrm{n}=18)$ \\
\hline Age (years) & $71(55-77)$ \\
\hline SAPS II (points) & $57(43-64)$ \\
\hline \multicolumn{2}{|l|}{ Main reason for $\mathrm{ICU}$ admission } \\
\hline Severe Sepsis & $15(83 \%)$ \\
\hline Major Surgery & $2(12 \%)$ \\
\hline Hemorrhagic Shock & $1(5 \%)$ \\
\hline \multicolumn{2}{|l|}{ Precipitating Factors } \\
\hline Cancer & $18(100 \%)$ \\
\hline Severe Sepsis & $18(100 \%)$ \\
\hline Major Surgery & $6(33 \%)$ \\
\hline Hemorrhagic Shock & $1(5 \%)$ \\
\hline HIV infection & $1(5 \%)$ \\
\hline \multicolumn{2}{|l|}{ Thrombotic/Ischemic events } \\
\hline Acrocyanosis & $18(100 \%)$ \\
\hline Skin necrosis (including surgical flaps) & $9(50 \%)$ \\
\hline Deep venous thrombosis & $5(28 \%)$ \\
\hline Arterial thrombosis & $9(50 \%)$ \\
\hline Cerebrovascular ischemia & $5(28 \%)$ \\
\hline Gastric ischemia & $5(28 \%)$ \\
\hline Colonic ischemia & $2(11 \%)$ \\
\hline Splenic infarction & $1(5 \%)$ \\
\hline
\end{tabular}

SAPSII=Simplified Acute Physiology Score; ICU=intensive care unit. Results are presented as median (25\%-75\% interquartile range) or numbers (percentage). 
Besides cancer, the main factors present were severe sepsis (18/18), major surgery (6/18), hemorrhagic shock (1/18) and HIV infection (1/18). aPL antibodies and coagulation tests were obtained 5 days (range: $3-12$ ) after ICU admission and only one patient had a previous thrombotic event. All patients had positive LAC and two patients had high titers of aCL antibodies. The most frequent thrombotic and ischemic events are listed in Table 1. Interestingly, despite the presence of abnormal coagulation parameters in all patients, most of them (10/18) did not fulfill the International Society of Hemostasis and Thrombosis (ISTH) criteria for overt disseminated intravascular coagulation (DIC), ${ }^{12}$ or they otherwise exhibited normal or near-normal protein $\mathrm{C}$ activity levels (Table 2 ). When the diagnostic criteria were applied, 16 patients met the diagnostic criteria for "probable" CAPS and two had definite CAPS., ${ }^{41}$ No patient had a previous diagnosis of aPL syndrome.

Table 2 - Laboratory parameters in critically ill cancer patients with antiphospholipid syndrome

\begin{tabular}{lc}
\hline Laboratory Data & \\
Positive Lupus anticoagulant antibody & $18(100 \%)$ \\
Positive Anticardiolipin antibody & $2(11 \%)$ \\
D-dimer (units/dL) & $794(271-1471)$ \\
Protein C (activity, \%) & $66,5(44-96)$ \\
Antithrombin (activity, \%) & $51(34-65)$ \\
Factor VIII (activity, \%) & $241(198-295)$ \\
Platelet count $\left(x ~ 10^{3} / \mathrm{mm}^{3}\right)$ & $122(73-199)$ \\
Fibrinogen (mg/dL) & $348(235-557)$ \\
\hline
\end{tabular}

Results are presented as median (25\%-75\% interquartile range) or numbers (percentage).

MOF was present in all patients. Acute lung injury and acute respiratory distress syndrome (18/18), cardiovascular collapse (18/18) and acute renal failure requiring dialysis (13/18) were prominent features. Despite the high prevalence of shock, only two patients were using extremely high doses $(>1.0 \mathrm{mcg} / \mathrm{Kg} / \mathrm{min})$ of norepinephrine at the occurrence of acrocyanosis/skin necrosis and no patient was administered epinephrine or vasopressin.

Treatments were administered on an individual patient basis, consistent with clinical assessments. Most patients $(16 / 18)$ were treated with systemic anticoagulation using unfractioned heparin. Although heparin infusion did not seem to impact global mortality, at least four patients that received early anticoagulation exhibited a reversal of the acrocyanosis and no further major vessel thrombotic events. Five patients were treated with plasmapheresis. Apart from corticosteroids, used in low doses for sepsis, only one patient received high doses $(1 \mathrm{~g})$ of methylprednisolone for three consecutive days.

The ICU, hospital and 90-day mortality rates were $61 \%$, $72 \%$ and $83 \%$, respectively. At 90-day follow up, only three patients were alive. New measurements of the LAC antibodies were performed in these three survivors and one patient tested positive for the presence of these antibodies.

\section{DISCUSSION}

In the present study we describe a small series of critically ill cancer patients who presented with apL antibodies, severe life-threatening thrombotic manifestations and multi-organ failure. Although the association between cancer and aPL antibodies is well-understood, the pathophysiological role of the aPL antibodies is less clear. Indeed, while some authors argue that these antibodies may be of pathological significance, others consider them an epiphenomenon of the malignancy. In fact, the notion that cancer patients positive for aPL antibodies are at high risk for thrombosis and have worse prognoses has only recently been introduced. ${ }^{2,3}$ Moreover, a recent hypothesis postulates that even transient aPL antibodies may be implicated in the pathogenesis of thrombosis. ${ }^{13}$ Only two studies addressed the issue of LAC antibodies in critically ill patients. ${ }^{14,15}$ Wenzel et al., in an elegant study with 51 patients (only five of whom had cancer), did not observe any significant differences in outcomes regarding patients' LAC antibody status after ICU admission. ${ }^{14}$ Sepsis and vasopressor support were frequently associated with LAC antibodies. However, no significant thrombotic events were observed during the study. This suggests that either these transient antibodies are associated with low pathogenic potential or that the study was insufficiently powered to detect thrombotic events. In a selected population of ICU patients, Aldawood et al. reported a frequency of $8 \%$ for transient LAC antibodies being associated with sepsis and mortality. However, they found no association between the presence of antibodies and major thrombotic events. ${ }^{15}$ Once again, very few cancer patients were evaluated and patients with hematologic malignancies were excluded. Heterogeneous mechanisms of coagulation are responsible for the increased risk of thrombosis in patients with cancer. ${ }^{16}$ Sepsis and SIRS have undisputable roles in the activation of coagulation, the development and amplification of endothelial activation, and microvascular thrombosis[8]. Therefore, it is plausible to hypothesize that severe pro-inflammatory and pro-coagulant insults such as severe sepsis may trigger catastrophic vascular events, especially in susceptible patients, such as those with cancer and positive aPL antibodies. ${ }^{4,5}$

The presence of acrocyanosis is a frequent finding in 
critically ill patients, particularly in those with septic shock, DIC or those requiring high doses of vasopressors. In this setting, acrocyanosis, skin necrosis, limb ischemia and thrombosis are often ascribed to micro- and macro-vascular occlusion. The criteria for overt DIC were present in only eight of our patients, and abnormalities in the anticoagulant protein C system were not observed in most patients (11/18). This not only implies that the vascular phenomena may be related to the aPL syndrome, but that the syndrome might have contributed to the development of multi-organ failure.

We acknowledge there our study exhibits certain important shortcomings. We note the small sample size and the retrospective design. However, these are common flaws of studies that aim to evaluate rare diseases or unusual events. Ours was a retrospective and descriptive study and it was not designed to describe the frequency of thrombosis or aPL syndrome in critically ill cancer patients. Thus, selection bias cannot be ruled out. Patients were identified and included in the study because they presented clinical signs of severe thrombotic events. Also, the present study does not allow us to prove a causal relation between the presence of aPL antibodies and either vascular or systemic events. Nevertheless, most patients (16/18) fulfilled the diagnostic criteria for "probable CAPS" and two had definite diagnoses. New tests are often required to establish whether the antibodies are permanent or transient, and Beta2 glycoprotein I antibodies should be measured in future studies for a more robust diagnosis. However, most patients did not survive the 12-week period to permit a second measurement of LAC antibodies. Of the five patients who survived until hospital discharge, two died in the weeks following and LAC antibodies continued to test positive in only one of the three 90-day survivors.

Those patients with negative antibodies had controlled cancer (lymphoma in remission and a resected colonic tumor), as opposed to the patient positive for antibodies who presented disease progression (rectal tumor) and was undergoing radiation therapy. Despite these limitations, we believe that the issue deserves attention and our investigation should be interpreted as a hypothesis-generating study.

In conclusion, CAPS may be more frequent than previously thought in critically ill cancer patients. In these patients, SIRS or sepsis coupled with aPL antibodies is associated with an exceedingly high number of thrombotic events, acute organ dysfunctions and an ominous prognosis. However, large prospective studies are mandatory to clearly establish the pathophysiological role and clinical relevance of aPL antibodies in critically ill cancer patients.

Financial support: institutional departmental funds.

\section{REFERENCES}

1. Miesbach W, Asherson RA, Cervera R, Shoenfeld Y, Gomez Puerta J, Bucciarelli S, et al. The catastrophic antiphospholipid (Asherson's) syndrome and malignancies. Autoimmun Rev. 2006;6:94-7.

2. Bairey O, Blickstein D, Monselise Y, Lahav J, Stark P, Prokocimer M, et al. Antiphospholipid antibodies may be a new prognostic parameter in aggressive non-Hodgkin's lymphoma. Eur J Haematol. 2006;76:38491.

3. De Meis E, Pinheiro VR, Loures MA, Kotouc MM, Zamboni MM, Guedes MT, et al. Lupus Anticoagulant Activity as a Thrombosis Risk Factor in Lung Adenocarcinoma Patients. Ann NY Acad Sci. 2007;1107:51-5.

4. Asherson RA. Multiorgan failure and antiphospholipid antibodies: the catastrophic antiphospholipid (Asherson's) syndrome. Immunobiology. 2005;210:727-33.

5. Shoenfeld Y, Blank M, Cervera R, Font J, Raschi E, Meroni PL. Infectious origin of the antiphospholipid syndrome. Ann Rheum Dis. 2006;65:2-6.

6. Berlin T, Zandman-Goddard G, Blank M, Matthias T, Pfeiffer S, Weis I, et al. Autoantibodies in nonautoimmune individuals during infections. Ann N Y Acad Sci. 2007;1108:584-93.

7. Soares M, Salluh JI, Spector N, Rocco JR. Characteristics and outcomes of cancer patients requiring mechanical ventilatory support for $>24 \mathrm{hrs}$. Crit Care Med. 2005;33:520-26.

8. Bakhtiari K, Meijers JC, de Jonge E, Levi M. Prospective validation of the International Society of Thrombosis and Haemostasis scoring system for disseminated intravascular coagulation. Crit Care Med. 2004;32:2416-21.

9. Le Gall JR, Lemeshow S, Saulnier F. A new Simplified Acute Physiology Score (SAPS II) based on a European/North American multicenter study. Jama. 1993;270:2957-63.
10. Bone RC, Balk RA, Cerra FB, Dellinger RP, Fein AM, Knaus WA, et al. Definitions for sepsis and organ failure and guidelines for the use of innovative therapies in sepsis. The ACCP/SCCM Consensus Conference Committee. American College of Chest Physicians/Society of Critical Care Medicine. Chest. 1992;101:1644-55.

11. Miyakis S, Lockshin MD, Atsumi T, Branch DW, Brey RL, Cervera R, et al. International consensus statement on an update of the classification criteria for definite antiphospholipid syndrome (APS). J Thromb Haemost. 2006;4:295-306.

12. Toh CH, Hoots WK. The scoring system of the Scientific and Standardisation Committee on Disseminated Intravascular Coagulation of the International Society on Thrombosis and Haemostasis: a 5-year overview. J Thromb Haemost. 2007;5:604-6.

13. Lim W, Crowther MA. Antiphospholipid antibiodies: a critical review of the literature. Curr Opin Hematol. 2007;14:494-9.

14. Wenzel C, Stoiser B, Locker GJ, Laczika K, Quehenberger P, Kapiotis $\mathrm{S}$, et al. Frequent development of lupus anticoagulants in critically ill patients treated under intensive care conditions. Crit Care Med. 2002;30:763-70.

15. Aldawood AS, Crowther M, Jaeschke R, Dabbagh O, Alkhairy K, Baharoon S, et al. The incidence and impact of lupus anticoagulants among patients in the intensive care unit. Saudi Med J. 2005;26:19945 .

16. Buller HR, van Doormaal FF, van Sluis GL, Kamphuisen PW. Cancer and thrombosis: from molecular mechanisms to clinical presentations. J Thromb Haemost 2007;5 Suppl 1:246-54. 